\title{
Had He Been Drinking or not?
}

DOI: $10.1134 / \mathrm{S} 1061934812020165$

In 2010, Nauka Publishing issued a voluminous monograph Vnelaboratornyi khimicheskii analiz. (Extralaboratory Chemical Analysis). Among its twenty-four chapters, is a one on the determination of ethanol in exhaled air. The problem is mainly the detection of alcohol vapors in the air exhaled by drivers.

Before 2008, in Russia, a "drunken state" could be diagnosed only by a doctor, based on a series of clinical symptoms, and the determination of alcohol concentration could only confirm the diagnosis. Even at a rather high concentration of alcohol in exhaled air, a doctor could conclude that the driver is not drunk if these clinical symptoms were not observed. The symptoms could not be detected because of the "tolerance" of some people to alcohol. However, a law that came in force in Russia in 2008 made an objective characteristic, alcohol concentration in exhaled air, the main one. The authors of the above chapter have written that, "In this connection, special requirements are put forward on the quality of measuring devices and the analysis procedure."

Appropriate methods and tools have been developed for several decades. Experienced drivers know tubes in which in the presence of alcohol in exhaled air is detected by green color. This is the MokhovShinkarenko tube and its modification, the so-called "sobriety control" tube. In these tubes, ethanol reduces potassium dichromate in the presence of concentrated sulfuric acid. Unfortunately, this method is not very selective: green color due to the reduction of chromium is also given by methanol, acetone, and certain other substances. If petrol vapors penetrate the tube, a brown coloring that masks the reaction with ethanol will be observed.

Many other devices have been proposed based on different principles. Of particular interest is the spectroscopic method based on infrared rays: ethanol selectively absorbs radiation with a wavelength of 3.4 and $9.5 \mu \mathrm{m}$. There are electrochemical devices. Some devices utilize two approaches, i.e., absorption of radiation at $9.5 \mu \mathrm{m}$ and electrochemical oxidation of ethanol to acetaldehyde, as in the German Alcotest 7110 instrument. Russia authorities use both foreign and domestic devices, manufactured by the Meta Corporation under the name AKPE-01.

Researchers of the Prokhorov General Physics Institute, Russian Academy of Sciences, have designed an instrument that allows the detection of alcohol vapors even in a moving car. It is based on diode lasers.

Recently in Russia, limits on alcohol use by drivers have been made much more stringent. These rates actually mean that alcohol concentration in exhaled air should be zero. Of course, this is hardly probable, because ethanol is present in a number of foodstuffs.

Yu. A. Zolotov 